PROCEEDINGS OF THE

AMERICAN MATHEMATICAL SOCIETY

Volume 126, Number 12, December 1998, Pages 3513-3519

S 0002-9939(98)05019-9

\title{
ON ONE SET OF ORTHOGONAL HARMONIC POLYNOMIALS
}

\author{
V. V. KARACHIK
}

(Communicated by J. Marshall Ash)

\begin{abstract}
A new basis of harmonic polynomials is given. Proposed polynomials are orthogonal on the unit sphere and each term of this basis consists of monomials not present in the others.
\end{abstract}

\section{INTRODUCTION}

For the investigation of harmonic polynomials a scalar product for homogeneous polynomials of degree $m$ in the form $\left\langle P_{m}(x), Q_{m}(x)\right\rangle=P_{m}(D) Q_{m}(x)$ was introduced in [1] - one of the basic works on harmonic analysis - where the operator $P_{m}(D)$ is obtained from the polynomial $P_{m}(x)$ by replacing each variable $x_{i}$ on the differential operator $\partial / \partial x_{i}$. If we denote a set of all polynomials over $C$ by $\mathcal{P}$, then that scalar product can be extended on $\mathcal{P}$ in the following way: $\langle P(x), Q(x)\rangle=P(D) Q(x)_{\mid x=0}$. This idea proved to be very successful for the investigation of polynomial solutions to systems of PDE with constant coefficients (see for instance [2], [3]). In the present work we shall consider a full set of harmonic polynomials which are furthermore orthogonal both in $L_{2}\left(\partial S_{n}\right)\left(S_{n}\right.$ is a unit ball in $R^{n}$ ) and in $\mathcal{P}$, and we shall give one interesting property of theirs (Corollary 2).

\section{System of harmonic Polynomials $G_{(\nu)}(x)$}

Let $k, s \in \widetilde{N}(\widetilde{N} \equiv N \cup\{0\})$ and $n \in N$ but $n>1$. Consider the polynomials

$$
G_{k}^{s}\left(x_{(n)}\right)=\sum_{i=0}^{[k / 2]}(-1)^{i} \frac{\left|x_{(n-1)}\right|^{2 i} x_{n}^{k-2 i, !}}{(2,2)_{i}(n-1+2 s, 2)_{i}},
$$

where $(a, b)_{i}=a(a+b) \ldots(a+i b-b)$ (with the convention $\left.(a, b)_{0}=1\right), x^{k, !}=x^{k} / k !$, and $x_{(n)}=\left(x_{1}, \ldots, x_{n}\right)$. We shall call them $G$-polynomials of degree $k$, order $s$, and kind $n$.

Received by the editors December 3, 1996.

1991 Mathematics Subject Classification. Primary 33D30; Secondary 33D25, 31B05.

Key words and phrases. Harmonic polynomials, orthogonality.

(C) 1998 American Mathematical Society 
If $n=2$, then there are only two linearly independent homogeneous harmonic polynomials of degree $k(k>0)$ and we can write them in the form

$$
H_{k}^{s}\left(x_{(2)}\right)=\sum_{i=0}^{[(k-s) / 2]}(-1)^{i} x_{1}^{2 i+s, !} x_{2}^{k-2 i-s, !}
$$

for $k \in N, s=0,1$, and with the convention $H_{0}^{0} \equiv 1$. It is not difficult to verify that $H_{k}^{s}\left(x_{(2)}\right)=G_{k-s}^{s}\left(x_{(2)}\right) x_{1}^{s}$.

Theorem 1. Let $n \geq 2$. The polynomials

$$
G_{(\nu)}\left(x_{(n)}\right)=G_{\nu_{1}-\nu_{2}}^{\nu_{2}}\left(x_{(n)}\right) \cdots G_{\nu_{n-1}-\nu_{n}}^{\nu_{n}}\left(x_{(2)}\right) x_{1}^{\nu_{n}},
$$

where $\nu \in \widetilde{N}^{n}, \nu_{1} \geq \cdots \geq \nu_{n}$, and $\nu_{n}=0,1$, make up a basis among homogeneous harmonic polynomials of degree $\nu_{1}$.

Remark. Polynomials $G_{(\nu)}$ can be determined also by the recurrence relation

$$
\begin{array}{ll}
G_{(\nu)}(x)=H_{\nu_{n-1}}^{\nu_{n}}(x), & n=2, \\
G_{(\nu)}\left(x_{(n)}\right)=G_{\nu_{1}-\nu_{2}}^{\nu_{2}}\left(x_{(n)}\right) G_{(\bar{\nu})}\left(x_{(n-1)}\right), & n>2,
\end{array}
$$

where $\bar{\nu}=\left(\nu_{2}, \ldots, \nu_{n}\right)$.

Proof. At first, for convenience, denote $k=\nu_{1}$ and $s=\nu_{2}$. We shall employ induction on the dimension $n$. If $n=2$, then $G_{(\nu)}\left(x_{(n)}\right)=G_{k-s}^{s}\left(x_{(2)}\right) x_{1}^{s}=H_{k}^{s}\left(x_{(2)}\right)$ and the theorem's statement is obviously true.

Let $n \geq 3$. Consider a set of homogeneous polynomials of degree $k$ having the form $v_{k}\left(x_{(n)}\right)=u_{s}\left(x_{(n-1)}\right) G_{k-s}^{s}\left(x_{(n)}\right)$, where $k$ is fixed, $s=0, \ldots, k$, and the polynomials $u_{s}\left(x_{(n-1)}\right)$ make up a basis among homogeneous harmonic (of $n-1$ variables) polynomials of degree $s$, which according to the induction hypothesis can be taken in the form (1) for $n=n-1$.

Let the vector $\mu$ correspond to some polynomial $u_{s}\left(x_{(n-1)}\right)$. If we denote $\nu=$ $\left(k, \mu_{1}, \ldots, \mu_{n-1}\right)$, then the polynomial $v_{k}\left(x_{(n)}\right)$ is of the form (1).

In a straightforward way from the Laplace equation we can verify that for $s \leq k$ the polynomials $v_{k}\left(x_{(n)}\right)$ are harmonic.

It is not hard to see that the polynomials of the form $v_{k}\left(x_{(n)}\right)$ are linearly independent. Indeed, if $s$ is fixed they are linearly independent because of the linear independence of $u_{s}\left(x_{(n-1)}\right)$, if $s=0, \ldots, k$ they have different degrees with respect to $x_{n}$ and therefore are linearly independent also.

Finally, we need to count the number of $v_{k}\left(x_{(n)}\right)$. It is known [1] that the number of $u_{s}\left(x_{(n-1)}\right)$ for $s>1$ is

$$
h_{s}^{n-1}=\left(\begin{array}{c}
s+n-2 \\
n-2
\end{array}\right)-\left(\begin{array}{c}
s+n-4 \\
n-2
\end{array}\right)
$$

and $h_{1}^{n-1}=n-1, h_{0}^{n-1}=1$. Therefore the number of $v_{k}\left(x_{(n)}\right)$ will be

$$
\sum_{s=2}^{k}\left(\left(\begin{array}{c}
s+n-2 \\
n-2
\end{array}\right)-\left(\begin{array}{c}
s+n-4 \\
n-2
\end{array}\right)\right)+n .
$$

After some transformations this number becomes equal to $h_{k}^{n}$. This ends the proof. 
Corollary 1. Let $\mathcal{G}_{n-1}$ be a basis in harmonic polynomials of $n-1$ variables. Then

$$
\mathcal{G}_{n}=\left\{G_{k}^{s}\left(x_{(n)}\right) u_{s}\left(x_{(n-1)}\right) \mid k \in \widetilde{N}, u_{s} \in \mathcal{G}_{n-1}\right\}
$$

is a basis in harmonic polynomials of $n$ variables.

We can easily extract the proof of this statement from the previous theorem.

In what follows, if no confusion will result, instead of $x_{(n)}$ and $x_{(n-1)}$ we shall write $x$ and $\tilde{x}$ respectively. Denote a square of $\partial S_{n}$ by $\omega_{n}$ and the norm of $u \in$ $L_{2}\left(\partial S_{n}\right)$ by $\|u\|$.

Lemma 1. Let $f \in C\left(\partial S_{n}\right)$ be taken in the form $f(x)=\varphi\left(|\tilde{x}|, x_{n}\right) P_{k}(\tilde{x})$, where $P_{k}(\tilde{x})$ is a homogeneous polynomial of degree $k$ and $\varphi \in C\left(\partial S_{n}\right)$. Then

$$
\int_{|x|=1} f(x) d x=\frac{1}{\omega_{n-1}} \int_{|x|=1} \varphi\left(|\tilde{x}|, x_{n}\right)\left(1-x_{n}^{2}\right)^{k / 2} d x \int_{|\tilde{x}|=1} P_{k}(\tilde{x}) d \tilde{x} .
$$

Proof. We can lightly verify that for $f \in C\left(\partial S_{n}\right)$ the following equality holds:

$$
\int_{|x|=1} f(x) d x=\int_{|\tilde{x}|<1}\left[f\left(\tilde{x}, \sqrt{1-|\tilde{x}|^{2}}\right)+f\left(\tilde{x},-\sqrt{1-|\tilde{x}|^{2}}\right)\right]\left(1-|\tilde{x}|^{2}\right)^{-1 / 2} d \tilde{x} .
$$

Further, making some transformations we get

$$
\int_{|x|=1} f(x) d x=\int_{0}^{1} \frac{\tau^{n-2}}{\sqrt{1-\tau^{2}}} d \tau \int_{|\tilde{x}|=1}\left[f\left(\tau \tilde{x}, \sqrt{1-\tau^{2}}\right)+f\left(\tau \tilde{x},-\sqrt{1-\tau^{2}}\right)\right] d \tilde{x} .
$$

If we make the substitution $t=\sqrt{1-\tau^{2}}$, then this yields

$$
\begin{aligned}
\int_{|x|=1} f(x) d x= & \int_{0}^{1}\left(1-t^{2}\right)^{(n-3) / 2} d t \\
& \cdot \int_{|\tilde{x}|=1}\left[f\left(\sqrt{1-t^{2}} \tilde{x}, t\right)+f\left(\sqrt{1-t^{2}} \tilde{x},-t\right)\right] d \tilde{x} .
\end{aligned}
$$

Dividing the integral on the right into two parts and substituting $t$ for $-t$ in the second integral we get

$$
\int_{|x|=1} f(x) d x=\int_{-1}^{1}\left(1-t^{2}\right)^{(n-3) / 2} d t \int_{|\tilde{x}|=1} f\left(\sqrt{1-t^{2}} \tilde{x}, t\right) d \tilde{x} .
$$

Now it is time to use the form of $f(x)$. We have

$$
\int_{|x|=1} f(x) d x=\int_{-1}^{1}\left(\sqrt{1-t^{2}}\right)^{n+k-3} \varphi\left(\sqrt{1-t^{2}}, t\right) d t \int_{|\tilde{x}|=1} P_{k}(\tilde{x}) d \tilde{x} .
$$

If we multiply this equality by

$$
\omega_{n-1}=\int_{|\tilde{x}|=1}|\tilde{x}|^{k} d \tilde{x}
$$

and use again the equality (3) with the function $f(x)=\varphi\left(|\tilde{x}|, x_{n}\right)|\tilde{x}|^{k}$, then we get

$$
\omega_{n-1} \int_{|x|=1} f(x) d x=\int_{|x|=1} \varphi\left(|\tilde{x}|, x_{n}\right)\left(1-x_{n}^{2}\right)^{k / 2} d x \int_{|\tilde{x}|=1} P_{k}(\tilde{x}) d \tilde{x},
$$

which implies (2). The proof is complete. 
Lemma 2. G-polynomials of the same order $s$ and kind $n$ are orthogonal with weight $\rho_{n}^{s}(x)=\left(1-x_{n}^{2}\right)^{s}$ on $\partial S_{n}$.

Proof. Let $G_{p}^{s}(x)$ and $G_{q}^{s}(x)(p \neq q)$ be two arbitrary $G$-polynomials of the same kind $n$, and let $u_{s}(\tilde{x})$ be any homogeneous harmonic polynomial of degree $s$. If we denote $u_{p+s}(x)=G_{p}^{s}(x) u_{s}(\tilde{x})$ and $u_{q+s}(x)=G_{q}^{s}(x) u_{s}(\tilde{x})$ respectively, then the polynomials $u_{p+s}(x)$ and $u_{q+s}(x)$ are, according to Corollary 1 , harmonic. Let us take advantage of Lemma 1 for the function $\varphi(x)=G_{p}^{s}(x) G_{q}^{s}(x)$ and the polynomial $P_{k}(\tilde{x})=u_{s}^{2}(\tilde{x})$. This is possible because $G_{k}^{s}(x)=G_{k}^{s}\left(|\tilde{x}|, x_{n}\right)$ and Lemma 1's condition is fulfilled. We have

$$
\int_{|x|=1} u_{p+s}(x) u_{q+s}(x) d x=\frac{1}{\omega_{n-1}} \int_{|x|=1} \rho_{n}^{s}(x) G_{p}^{s}(x) G_{q}^{s}(x) d x \int_{|\tilde{x}|=1} u_{s}^{2}(\tilde{x}) d \tilde{x} .
$$

Since the left-hand side of this equality vanishes $(p+s \neq q+s)[1]$ and $\left\|u_{s}\right\| \neq 0$ we arrive at the desired result. The proof is complete.

Now we are able to establish an orthogonality of the system $\left\{G_{(\nu)}\right\}$ on $\partial S_{n}$.

Theorem 2. Polynomials $G_{(\nu)}(x)$ for any distinct vectors $\nu \in \widetilde{N}^{n}$ satisfying the condition $\nu_{1} \geq \cdots \geq \nu_{n}\left(\nu_{n}=0,1\right)$ are orthogonal on $\partial S_{n}$.

Proof. In just the same way as in Theorem 1 we employ the induction on the dimension $n$. Let $n=2$ and $\nu=(m, s)$. For $x \in \partial S_{2}$ the polynomials $G_{(\nu)}(x)=$ $H_{m}^{s}(x)$ can be represented in the form

$$
H_{m}^{0}(x)=\frac{1}{m !} \cos \left(m \arccos x_{2}\right), \quad H_{m}^{1}(x)=\frac{1}{m !} \sin \left(m \arccos x_{2}\right),
$$

where $x_{2} \in[-1,1]$ and therefore are orthogonal on $\partial S_{2}$.

Let $n \geq 3$ and $\nu, \mu$ be two unequal vectors from $\widetilde{N}^{n}$ satisfying the theorem's condition. Let us take advantage of Lemma 1 keeping in mind the Remark to Theorem 1. We have

$$
\int_{|x|=1} G_{(\nu)}(x) G_{(\mu)}(x) d x=
$$

$$
\frac{1}{\omega_{n-1}} \int_{|x|=1} G_{\nu_{1}-\nu_{2}}^{\nu_{2}}(x) G_{\mu_{1}-\mu_{2}}^{\mu_{2}}(x)\left(1-x_{n}^{2}\right)^{\left(\nu_{2}+\mu_{2}\right) / 2} d x \int_{|\tilde{x}|=1} G_{(\bar{\nu})}(\tilde{x}) G_{(\bar{\mu})}(\tilde{x}) d \tilde{x} .
$$

There are only two possibilities: either $\nu_{1} \neq \mu_{1}, \bar{\nu}=\bar{\mu}$ or $\nu_{1}=\mu_{1}, \bar{\nu} \neq \bar{\mu}$. In the first case the right-hand side of (4) vanishes by virtue of Lemma $2\left(\nu_{1} \neq \mu_{1}\right.$, $\left.\nu_{2}=\mu_{2} \Rightarrow \nu_{1}-\nu_{2} \neq \mu_{1}-\mu_{2}\right)$ and in the second case, by virtue of the induction hypothesis. The proof is complete.

Theorem 3. Polynomials $G_{(\nu)}(x)$ for any distinct vectors $\nu \in \widetilde{N}^{n}$ satisfying the condition $\nu_{1} \geq \cdots \geq \nu_{n}\left(\nu_{n}=0,1\right)$ are orthogonal in $\mathcal{P}$.

Proof. We need to check the correctness of the following statement: $\nu \neq \mu \Rightarrow$ $\left\langle G_{(\nu)}, G_{(\mu)}\right\rangle=0$. To do this we are going to employ again the induction on the dimension $n$. We shall consider the case $\nu_{1}=\mu_{1}$ because if $\nu_{1} \neq \mu_{1}$, then the polynomials $G_{(\nu)}(x)$ and $G_{(\mu)}(x)$ are orthogonal as having different degree.

Let $n=2$. The polynomials $G_{(\nu)}(x)$ and $G_{(\mu)}(x)$ are orthogonal because they have different evenness with respect to $x_{1}$ : if $\nu_{2}=0$, then $G_{(\nu)}(x)$ is even, and if $\nu_{2}=1$, it is odd. 
Let $n>2$. Consider the case $\nu_{2}<\mu_{2}$. Determine $G$-polynomials for $k \in Z \backslash \widetilde{N}$ as $G_{k}^{s} \equiv 0$ and denote $\widetilde{\Delta}=\Delta-\partial^{2} / \partial x_{n}^{2}$. It is not difficult to verify that

$$
\frac{\partial}{\partial x_{n}} G_{(\mu)}(x)=G_{\left(\mu_{-}\right)}(x), \quad \widetilde{\Delta} G_{(\mu)}(x)=-G_{\left(\mu_{=}\right)}(x),
$$

where $\mu_{-}=\left(\mu_{1}-1, \mu_{2} \ldots, \mu_{n}\right)$ and $\mu_{=}=\left(\mu_{-}\right)_{-}$. Therefore using the Remark to Theorem 1 we can get

$$
G_{(\nu)}(D) G_{(\mu)}(x)=C G_{(\bar{\nu})}(\widetilde{D})\left[G_{\left(\mu_{1}-\mu_{2}\right)-\left(\nu_{1}-\nu_{2}\right)}^{\mu_{2}}(x) G_{(\bar{\nu})}(\tilde{x})\right],
$$

where

$$
C=\sum_{i=0}^{\left[\nu_{1} / 2\right]} \frac{1}{(2,2)_{i}\left(n-1+2 \nu_{2}, 2\right)_{i}\left(\nu_{1}-2 i\right) !} .
$$

Since $\mu_{1}-\mu_{2}-\nu_{1}+\nu_{2}=\nu_{2}-\mu_{2}<0$ we can conclude that $G_{(\nu)}(x)$ and $G_{(\mu)}(x)$ are orthogonal. The same is true for $\nu_{2}>\mu_{2}$ also. Consider the last case $\nu_{2}=\mu_{2}$. Using the previous equality we obtain $\left\langle G_{(\nu)}, G_{(\mu)}\right\rangle=C\left\langle G_{(\bar{\nu})}, G_{(\bar{\mu})}\right\rangle$. Since $\nu_{1}=\mu_{1}$ and $\nu \neq \mu$ we have $\bar{\nu} \neq \bar{\mu}$. Hence, by the induction hypothesis $G_{(\nu)}(x)$ and $G_{(\mu)}(x)$ are orthogonal. The proof is complete.

\section{Property of the polynomials $G_{(\nu)}(x)$}

Consider the polynomials $\bar{G}_{(\nu)}(x)$ to be obtained from $G_{(\nu)}(x)$ by normalization in $L_{2}\left(\partial S_{n}\right)$. In what follows without stipulation we shall use $\nu$ as a vector from $\widetilde{N}^{n}$ satisfying the condition $\nu_{1} \geq \cdots \geq \nu_{n}\left(\nu_{n}=0,1\right)$. For $n>2$ denote

$$
E(x, \xi)=\frac{1}{(n-2) \omega_{n}}|x-\xi|^{2-n} .
$$

Lemma 3. If $|x|<|\xi|$, then

$$
E(x, \xi)=\sum_{\nu} \frac{|\xi|^{-\left(2 \nu_{1}+n-2\right)}}{2 \nu_{1}+n-2} \bar{G}_{(\nu)}(x) \bar{G}_{(\nu)}(\xi) .
$$

Proof. Let $C_{k}^{l}[t]$ be Gegenbauer's polynomial [4]. So if $|\xi|=1$ and $|x|<1$ we have

$$
|x-\xi|^{2-n}=\sum_{k=0}^{\infty} C_{k}^{n / 2-1}\left[\left(\frac{x}{|x|}, \xi\right)\right]|x|^{k} .
$$

According to [4] for any $k \in \widetilde{N}$ and $\xi, \eta \in \partial S_{n}$ we can get the equality

$$
\frac{1}{\omega_{n}} \frac{C_{k}^{n / 2-1}[(\xi, \eta)]}{C_{k}^{n / 2-1}[1]}=\frac{1}{h_{k}^{n}} \sum_{\nu_{1}=k} \bar{G}_{(\nu)}(\xi) \bar{G}_{(\nu)}(\eta),
$$

where $h_{k}^{n}$ is defined in Theorem 1, summation is taken over all $\nu$ such that $\nu_{1}=k$, and $C_{k}^{n / 2-1}[1]=(k+n-3) ! /(k !(n-3) !)$. After using this expression in (6) we obtain

$$
\frac{1}{\omega_{n}}|x-\xi|^{2-n}=\sum_{k=0}^{\infty} \frac{n-2}{2 k+n-2} \sum_{\nu_{1}=k} \bar{G}_{(\nu)}(x) \bar{G}_{(\nu)}(\xi) .
$$

If now observe that $E(x, \xi)=|\xi|^{2-n} E(x /|\xi|, \xi /|\xi|)$, where $|x /| \xi||<1$, then (7) can be easily transformed to the form (5). The proof is complete. 
Lemma 4. Let $\xi, \eta \in \partial S_{n}$ and $k \in \tilde{N}$. Then

$$
\frac{1}{h_{k}^{n}} \sum_{\nu_{1}=k}\left|\bar{G}_{(\nu)}(\xi) \bar{G}_{(\nu)}(\eta)\right| \leq \frac{1}{\omega_{n}} .
$$

Proof. Plugging $|\xi|=1$ and $x=\varepsilon \xi$ into (5), for $\varepsilon \in(0,1)$ we get

$$
\frac{1}{(n-2) \omega_{n}}(1-\varepsilon)^{2-n}=\sum_{k=0}^{\infty} \frac{\varepsilon^{k}}{2 k+n-2} \sum_{\nu_{1}=k}\left(\bar{G}_{(\nu)}(\xi)\right)^{2} .
$$

Hence, keeping in mind that

$$
(1-\varepsilon)^{2-n}=\sum_{k=0}^{\infty}\left(\begin{array}{c}
k+n-3 \\
n-3
\end{array}\right) \varepsilon^{k}
$$

we obtain

$$
\sum_{\nu_{1}=k}\left(\bar{G}_{(\nu)}(\xi)\right)^{2}=\frac{1}{\omega_{n}} \frac{2 k+n-3}{n-2}\left(\begin{array}{c}
k+n-3 \\
n-3
\end{array}\right)=\frac{h_{k}^{n}}{\omega_{n}} .
$$

To get the desired estimate (8) it is sufficient to use the inequality

$$
2\left|\bar{G}_{(\nu)}(\xi) \bar{G}_{(\nu)}(\eta)\right| \leq \bar{G}_{(\nu)}^{2}(\xi)+\bar{G}_{(\nu)}^{2}(\eta) .
$$

This ends the proof.

Theorem 4. Let $u(x)$ be a harmonic function in $S_{n}$ and a continuous one in $\bar{S}_{n}$. Then for $x \in S_{n}$ the expansion

$$
u(x)=\sum_{\nu} u_{\nu} \bar{G}_{(\nu)}(x),
$$

where $u_{\nu}$ are Fourier coefficients of $u(x)$ by the system $\left\{\bar{G}_{(\nu)}\right\}$, is true and we can differentiate it any time under the summation sign.

Proof. It is not hard to verify that for $x \neq \xi$ and $\Lambda=x_{1} \partial / \partial x_{1}+\cdots+x_{n} \partial / \partial x_{n}$ we have

$$
\frac{1}{\omega_{n}} \frac{1-|x|^{2}}{|x-\xi|^{n}}=\Lambda_{x} E(x, \xi)-\Lambda_{\xi} E(x, \xi),
$$

and hence for $|x|<|\xi|=1$, according to Lemma 3, we obtain

$$
\frac{1}{\omega_{n}} \frac{1-|x|^{2}}{|x-\xi|^{n}}=\sum_{\nu} \bar{G}_{(\nu)}(x) \bar{G}_{(\nu)}(\xi) .
$$

Differentiation and passage to the limit under the summation sign is valid because, by virtue of Lemma 4, the series in (10) is uniformly convergent with respect to both $\xi \in \bar{S}_{n}$ and $|x| \leq \alpha<1$. Plugging (10) into the Poisson formula for the Dirichlet problem in $S_{n}$ we arrive at (9). Differentiation under the summation sign in (9) is valid also by virtue of Lemma 4 .

Let $P(x)$ be obtained from $\mathcal{P}$; then denote by $|P|_{D}$ its norm in $\mathcal{P}$.

Corollary 2. Let $u(x)$ be a harmonic function in $S_{n}$ and a continuous one in $\bar{S}_{n}$. Then

$$
G_{(\nu)}(D) u(x)_{\mid x=0}=g_{\nu} \int_{|\xi|=1} G_{(\nu)}(\xi) u(\xi) d \xi,
$$

where $g_{\nu}=\left|G_{(\nu)}\right|_{D}^{2} /\left\|G_{(\nu)}\right\|^{2}$. 
Proof. Consider the equality (9). Applying to it the operator $G_{(\mu)}(D)$ and setting $x=0$ we obtain

$$
G_{(\mu)}(D) u(x)_{\mid x=0}=\sum_{\nu_{1}=\mu_{1}} \frac{\left\langle G_{(\mu)}, G_{(\nu)}\right\rangle}{\left\|G_{(\nu)}\right\|^{2}} \int_{|\xi|=1} G_{(\nu)}(\xi) u(\xi) d \xi .
$$

If we now use Theorem 3 , then we immediately get (11). The proof is complete.

Example. Let $u(x)$ be as described in Corollary 2. Then

$$
\begin{gathered}
\frac{1}{\omega_{n}} \int_{|\xi|=1} \xi_{i} u(\xi) d \xi=\frac{1}{n} u_{x_{i}}(0), \quad \frac{1}{\omega_{n}} \int_{|\xi|=1} \xi_{i} \xi_{j} u(\xi) d \xi=\frac{1}{n(n+2)} u_{x_{i} x_{j}}(0), \\
\frac{1}{\omega_{n}} \int_{|\xi|=1} \xi_{i}^{2} u(\xi) d \xi=\frac{1}{n(n+2)} u_{x_{i} x_{i}}(0)+\frac{1}{n} u(0) .
\end{gathered}
$$

These equalities can be easily obtained from (11) if we take $G_{(\nu)}(x)=x_{i}$ (when $\nu=e_{1}+\cdots+e_{n-i+1}$, where $\left.e_{i}=\left(\delta_{1, i}, \ldots, \delta_{n, i}\right)\right), G_{(\nu)}(x)=x_{i} x_{j}$ (when $\nu=$ $2 e_{1}+\cdots+2 e_{n-j+1}+e_{n-j+2}+\cdots+e_{n-i+1}$ if $\left.i>j\right)$, and $G_{(\nu)}(x)=x_{n}^{2} / 2-|\tilde{x}|^{2} /(2 n-2)$ (when $\nu=2 e_{1}$ ) respectively.

\section{REFERENCES}

1. E.M.Stein and G.Weiss, Introduction to Fourier Analysis on Euclidian Spaces, Princeton Univ. Press, Princeton, NJ, 1971. MR 46:4102

2. V.V.Karachik, O polinomialnyh reshenijah sistem linejnyh differenzialnyh uravnenij, Voprosi Vychislitelnoy i prikladnoy matematiki 82 (1987), 41-48 (Russian).

3. P.Pedersen, A basis for polynomial solutions to the systems of linear constant coefficient PDE's, Advances Math., Article No.0005 117 (1996), 157-163. MR 96k:35018

4. H.Bateman and A.Erdélyi, Higher Transcendental Functions, vol. 2, New York, 1953.

Institute of Cybernetics of Academy of Science of Uzbekistan, 34, F.Hodzhaev St., TASHKENT, 700143, UZBEKISTAN

E-mail address: karachik@uwed.freenet.uz 